\title{
A Pot Ignored Boils On: Sustained Calls for Explicit Consent of Intimate Medical Exams
}

\author{
Lori Bruce $^{1}$ (i)
}

Published online: 9 March 2020

(c) Springer Nature B.V. 2020

\begin{abstract}
Unconsented intimate exams (UIEs) on men and women are known to occur for training purposes and diagnostic reasons, mostly during gynecological surgeries but also during prostate examinations and abdominal surgeries. UIEs most often occur on anesthetized patients but have also been reported on conscious patients. Over the last 30 years, several parties - both within and external to medicine-have increasingly voiced opposition to these exams. Arguments from medical associations, legal scholars, ethicists, nurses, and some physicians have not compelled meaningful institutional change. Opposition is escalating in the form of legislative bans and whistleblower reports. Aspiring to professional and scientific detachment, institutional consent policies make no distinction between intimate exams and exams on any other body part, but patients do not think of their intimate regions in a detached or neutral way and believe intimate exams call for special protections. UIEs are found to contribute to moral erosion and moral distress of medical students and compromise the sacred trust between the medical community and the general public. This paper refutes the main arguments in favor of the status quo, identifies a series of harms related to continuing the current practice, and proposes an explicit consent policy for intimate exams along with specific changes to medical school curriculum and institutional culture. Because patients are the rights-holders of their bodies, consent practices should reflect and uphold patient values which call for explicit consent for intimate exams.
\end{abstract}

Keywords Pelvic exams $\cdot$ Rectal exams $\cdot$ Unconsented $\cdot$ Informed consent $\cdot$ Medical education $\cdot$ Trust $\cdot$ Autonomy

Lori Bruce

Lori.Bruce@Yale.edu

1 Interdisciplinary Center for Bioethics, Yale University, New Haven, USA 


\section{Introduction}

From Hippocrates to Percival, classic medical scholars demonstrated "virtually no appreciation of a patient's right to consent" (Beauchamp 2011, p. 515). They avoided disclosures perceived to "harm or upset patients" and viewed patients' bodies as vessels for research and physician education; a view not to be superseded by a patient's preferences (Beauchamp 2011). Prioritizing research and education over a patient's preferences implies utilitarianism, when the goodness of an action is rooted in creating the most positive outcome for the greatest number of people. With the advent of bioethics, clinical medicine moved to a deontological frame in which the goodness of an action is based on whether that action in itself is right or wrong. Bioethics also phased out the idea that physicians' perceptions of a patient's best interests should supersede the patient's own values. Informed consent allows the patient to decide what happens to their body based on an understanding of the proposed procedure, and this concept was "imposed on medicine through nonmedical forms of authority such as judges in courts and government officials in regulatory agencies" (Beauchamp 2011, p. 515), underscoring the dichotomy between physicians' and nonphysicians' perceptions of rights.

Consent is now a well-established tenet of medical practice. Physicians must request a patient's permission before conducting research or performing procedures on them. Consent "must be rooted in autonomous choice by patients" (Beauchamp 2011, p. 518) and requires an explanation of the procedure and disclosure of the risks and benefits. Medicine is comprised of committed, talented individuals who dedicate their lives to healing the wounded and comforting the dying; however, certain practices still demonstrate concerning perceptions. Recent surveys and reports reveal that some physicians and medical students still conduct unconsented intimate exams (UIEs) on male and female patients. Unconsented rectal exams on men and women have been reported for training purposes (Reese and Monrouxe 2011; Coldicott et al. 2003a, b) and diagnostic reasons (Chan 2017). Unconsented vaginal exams have also been reported, mostly during gynecological surgeries (Schniederjan and Donovan 2005; Barnes 2012; Picard 2018) but also during abdominal surgeries (Graham 2019; Kelly 2019) and in efforts to rule out sexually transmitted disease in a patient with uncontrolled vomiting (Laird 2019). The referenced studies surveyed hundreds of medical students in the U.S., England, Wales, and Australia. In one study, nearly three-quarters of students believed that patients did not consent to their training exams (Schniederjan and Donovan 2005). In another study, patient consent for intimate exams was not acquired by half of the third-year students or one-third of patients examined by second-year students (Coldicott et al. 2003a, b).

Medical students are eager to participate in procedures and quickly realize the delicate balance of gaining new skills while respecting patient autonomy. Students are also careful to navigate complex organizational dynamics inherent to the academic culture of their medical schools. A student refusal to participate in an unconsented intimate exam (UIE) may be viewed as an act of personal 
embarrassment or unwarranted disobedience. Because students worry about jeopardizing their budding careers, they sometimes participate in clinical experiences that conflict with their personal moral compasses. Fraught dynamics between student and supervisor are central to the controversy of intimate exams on patients.

A pelvic exam is a standard precursor to gynecologic surgery to ascertain the position and mobility of the reproductive organs. The exam requires the insertion of one or two fingers into the vagina to palpate the ovaries and uterus. Rectal exams similarly require digital penetration with the aim of detecting abnormalities in the rectum, prostate, and abdomen. Medical schools consider these digital exams as standard training procedures and typically require all students to practice them during their third-year clerkships. Some institutions have policies in place requiring explicit consent for intimate exams, but they are the exception; most institutions have no such policies. Physicians often assume consent for these training exams, believing it is covered under general admissions clauses - or vague language on consent forms-relating to student participation in teaching hospitals. Consent forms often require the attending physician to hand-write the major risks of a specific procedure but otherwise follow a standard template. Patients and members of the general public are often shocked to learn that physicians and medical students may perform intimate exams on them without explicit consent and believe such exams meet the criteria for battery, malpractice, or sexual assault. Whether these exams meet any such criteria is contested, but this tension underscores the dichotomy between societal and medical views of the intimate regions of the body. While the medical professional may aspire to a professional, scientific detachment, patients simply do not think of their intimate regions in a detached or neutral way and tend to have an inherent conviction that consent practices should mark this distinction.

For several decades, ethicists and some number of physicians have expressed concern about UIEs. In 1989, Cohen et al. emphasized the uniqueness of the pelvic exam and how patients have a right to refuse participation in these training exams. Since then, UIEs continue while opposition increases. Singer considers UIEs "definitely among the most egregious of... ethical issues" in medical schools (Boyles 2003); Caplan (2018b) recently called for "an abrupt and immediate halt" to these exams; and Friesen (2018a) refers to UIEs as "immoral and indefensible" (p. 298). Adashi, a former dean at the Warren Alpert Medical School of Brown University, states, "viewed in hindsight, it is difficult to see how the conduct of unapproved pelvic examinations by medical students could have been rationalized, let alone condoned" (2019).

Medical students are increasingly raising concerns to their ethics advisors (Friesen 2018b; Caplan 2018a), researchers (Reese and Monrouxe 2011), the media (Tsai 2019), and state legislators (Barnes 2019). When medical students feel they must warn the public about UIEs in their medical institution, their actions meet the criteria for whistleblowing which:

results from a malfunction of the ethical environment of the organization to focus on its accountability for the safety and welfare of the patients. Individuals [who engage in whistleblowing] believe they must take a stand for the wrongdoing in the organization. (Lachman 2008, p. 265) 
Some of these whistleblower reports may have contributed to changes in association guidelines. Medical associations have developed consensus statements recommending bans on unconsented pelvic exams (Association of American Medical Colleges 2003; American College of Obstetricians and Gynecologists 2009); however, these statements are advisory and incomplete. Associations simply do not have the capacity to compel systemic change, as evidenced by institutions' inaction. In response, nine states recently passed legislation related to unconsented intimate exams, and other states are considering similar measures.

This paper describes current laws and association guidelines related to UIEs then explores the main arguments in favor of continuing the status quo. Next, the paper examines the implications of continuing the status quo and closes with a series of practical recommendations to curtail the occurrence of UIEs.

\section{Association Guidelines and Law}

The Association of American Medical Colleges (AAMC), American College of Obstetricians and Gynecologists (ACOG), and the American Medical Association (AMA) have called for explicit, mandatory consent before pelvic exams. The AAMC's statement (2003) declared, "medical students and residents not only must acquire clinical skills, they must also learn to always treat their patients with respect and dignity. Recent reports have suggested that medical students are performing pelvic examinations on women under anesthesia, without their knowledge and approval. AAMC believes that such practice is unethical and unacceptable" (2003). ACOG's 2011 opinion includes, "Pelvic examinations on an anesthetized woman that offer her no personal benefit and are performed solely for teaching purposes should be performed only with her specific informed consent obtained before her surgery" (p. 358). The AMA's Code of Medical Ethics Opinions on Patient-Physician Relationships states that patients' "refusal of care by a trainee should be respected in keeping with ethics guidance" (2001, p. 87), and a statement by the AMA Council on Ethical and Judicial Affairs calls for explicit disclosure of student involvement in exams on anesthetized patients (2001). Nurses have also expressed concern: fourteen nursing organizations banded together to urge the American Academy of Medical Colleges to require explicit consent (Wilson 2005).

The nine states who passed laws banning some form of intimate exams are Iowa, Illinois, Utah, Oregon, Maryland, Virginia, New York, California, and Hawaii. Other states are also considering or have proposed legislative action. Utah and Maryland's laws are gender-neutral but most other states explicitly specify unconsented pelvic exams on women. UIEs are also under scrutiny in other countries. New Zealand has a national consensus statement requiring written consent (Bagg et al. 2015), and France has taken steps to ban both unconsented rectal and pelvic exams (The Local 2015). Associations in other countries, including Canada (Liu et al. 2010) and the United Kingdom (Royal College of Obstetricians and Gynaecologists 2015), have explicit consent guidelines but they are limited to women's intimate exams. 


\section{Arguments}

As mentioned, medical associations, legislators, ethicists, and nurses-along with some physicians and medical students-have argued in favor of explicit consent of intimate exams. Arguments in favor of the status quo (which we define as failing to explicitly consent for intimate exams) are limited to those made by some physicians, institutions, and medical students. This section defines and discusses arguments in favor of the status quo.

\section{Explicit Consent Is Not Important}

Some medical students voice disinterest in the importance of consent (Cohen et al. 1988). One medical student called explicit consent an "attempt to justify the obsession with political correctness," opining that explicit consent is an inappropriate overreaction to a necessary training procedure (Bhangu 2003, p. 1326). Some physicians also find that explicit consent is simply not a priority. Citing his inclination as a "policy minimalist," George Washington University's OB-GYN chairman, John Larsen, expressed disinterest in codifying explicit consent (Goldstein 2003).

Interestingly, Ubel, a physician who examined UIE practices in teaching hospitals in a 2003 survey of 401 medical students, found that completion of the obstetrics/gynecology clerkship is associated with decreased interest in informed consent (Ubel 2003). After this clerkship, students were also "less likely to think consent for rectal examinations was important" (p. 578). In a follow-up interview, Ubel stated that the experience of participating in "exams without detailed consent on anesthetized patients desensitized doctors about the need for patients to grant consent" (Goldstein 2003). This view is in contrast with women who "almost universally [feel] that pelvic examinations that are conducted by medical students while the woman is anesthetized should occur only after the woman has given her permission to the students' supervisors" (Ubel 2003, p. 575).

To claim that explicit consent is unnecessary is in defiance of the fundamental principles of autonomy and self-determination. Adult patients have the right to self-determination: "the right to make the ultimate decision concerning what will or will not be done to their bodies" (Annas et al. 1977). Along with this right comes the right to refuse procedures. "Courts have... declared that both the common law and the United States Constitution protect an individual's right to refuse medical treatment" (Annas et al. 1983, p. 918). Similarly, the AMA's ethical and policy guidelines voice respect for the patient's refusal of an exam by a trainee (2016). The idea that explicit consent for intimate exams is optional therefore fails to meet the most basic tenets outlined in law, bioethics principles, and association guidelines. 


\section{Student Discomfort}

Another argument against explicit consent relates to discomfort felt by novice medical students when performing intimate exams. Such exams are "anxiety- or fear-provoking for the novice learner, [and they] often feel embarrassed or even uncomfortable" (Nelson 2016, p. 2). Some therefore say there is a benefit to UIEs because it is easier on the medical student to learn the exam while they do not have to consider student-patient dynamics. If the medical student makes a mistake or does something that would be physically or emotionally uncomfortable for the patient, the student will not be embarrassed by a conscious patient's reaction. However, there are several points against this argument.

First, teaching institutions have a duty to train their medical students, but training is secondary to their primary responsibility of the patient's health and well-being. Second, part of medical school is learning to exhibit professionalism with patients even when the student may be uncomfortable. Third, there are many ways of learning intimate exams to help students overcome their nerves before direct patient contact. Pressure-sensitive pelvic mannequins, training videos, and gynecologic teaching assistants (GTAs) contribute to comprehensive training. GTAs are women "trained on how to instruct the pelvic exam and allow students to perform the exam on them" (Nelson 2016, p. 3). GTAs are used in the U.S. as well as The Netherlands, Sweden, Belgium, Australia, and the UK (Walsh 2013).

Women with a history of sexual violence are at risk for pronounced fears, anxiety, pain, and discomfort during intimate exams (Qaseem et al. 2014), and it is reasonable to assume men with similar histories would have similar reactions. Other populations who experience pronounced levels of distress during intimate exams include people with disabilities, those who meet the criteria for obesity, and members of sexual and gender minorities (Potter 2015). In the U.S., the prevalence for sexual violence is $33 \%$ for women and $17 \%$ for men (National Sexual Violence Resource Center 2018); the prevalence for disabilities is approximately 20\% (Center for Disease Control and Prevention 2013); the prevalence of obesity is nearly $40 \%$ (Centers for Disease Control and Prevention 2016); and those who identify as LGBT + in the U.S. account for approximately $5 \%$ of the population (Family Equality Council 2017).

Medical students ought to develop sensitivity and understanding for these vulnerable populations which comprise a notable portion of the patient population. GTAs, sexual assault counselors, and experts in LGBT + ethics, and other experts can and should be consulted to help students learn to communicate sensitively with vulnerable patients during intimate exams. For instance, GTAs could expand their teaching to role-play as anxious patients so that students can practice their communication skills in a forgiving environment. Sexual assault counselors uniquely understand the trauma felt by survivors of sexual assault and can offer guidance in terms of vocabulary and alternative positioning of the patient body to more fully empower these patients. Experts in LGBT + ethics can similarly be consulted to help medical students more fully understand how current medical practice may marginalize these communities and how to more fully provide empathic care. 
Medical schools can carefully plan their teaching modules so that students can participate in these lower-stress learning experiences- and learn basic patient interaction skills and consent procedures-before they perform the exam on a patient. In short, there are obvious, uncomplicated means to help students overcome their nerves and increase competency, and student nervousness should not take priority over the right of a patient to decide what will happen to their body during a medical procedure.

\section{Intimate Exams are a Requisite Component of Standard Annual Exams}

Some argue that students must perform extensive numbers of intimate exams because the exams are a necessary component of regular patient care. However, in their Final Recommendation Statement: Screening for Prostate Cancer, the U.S. Preventive Services Task Force (USPSTF) recommends that men can either forgo a prostate exam or "make an individual decision about whether to be screened after a conversation with their clinician about the potential benefits and harms" (2018). Similarly, physicians are now questioning the "very utility of the traditional pelvic examination" (Adashi 2019). A recent study reviewed data from 2.6 million women aged 15-20 years old and found that $54.4 \%$ of their pelvic exams "were potentially unnecessary... and may cause harms such as false-positive test results, overdiagnosis, anxiety, and unnecessary costs" (Qin et al. 2020, p. E2). The American College of Obstetricians and Gynecologists advocates "against performing screening pelvic examinations in asymptomatic, nonpregnant, adult women" (2018, p. e174). Similarly, the USPSTF's Final Recommendation Statement for Gynecological Conditions states, "it is unclear whether performing screening pelvic examinations in asymptomatic women reduces morbidity and mortality" (2017). As medicine has evolved, the need for frequent, across-the-board digital intimate exams has lessened. This development especially decreases the need for frequent training exams by students in their clerkships.

\section{A Threat to Properly Training Physicians}

Some say that UIEs should continue because an explicit consent policy would substantially reduce the number of training exams, resulting in poorly trained physicians. In other words, if patients are asked, the belief is that they would likely refuse, so physicians may avoid the consent conversation so as not to jeopardize training opportunities. However, a recent survey of over 100 women indicated that $62 \%$ of women would consent if asked, and only $14 \%$ were sure they would refuse (Wainberg et al. 2010). Other surveys have similar positive feedback from patients related to their willingness to participate (Friesen 2018a), and students also have additional training opportunities in clinics and during training sessions with GTAs. In sum, explicit consent is an unlikely barrier to physician training. 


\section{Standard Surgical Consent Forms Are Good Enough}

Some physicians argue that explicit consent for intimate exams is unnecessary because intimate regions of the body are no different than any other body part. In response to Coldicott's landmark study on intimate exams (2003a, b), Kaushik, an ophthalmic surgeon, argues in favor of the status quo, believing it is "dangerous" to consider intimate exams differently, and that "examination of the fundus of the uterus is just as intimate for a gynaecologist [emphasis added] as examining the fundus of the eye is to an ophthalmologist" (2003a, b, p. 1327). This thinking contributes to physician preference for a standard, genericized surgical consent form for all surgeries. Yet other physicians understand that the frame of reference should not be about whether the physician finds the exam to be intimate. Rather, the decades-long conflict relating to UIEs relates to how these exams are perceived by the patients themselves.

Women's pelvic exams are "particularly threatening" to the patient (Cohen et al. 1988) and "in the area of gynecologic care [patients' right to refuse student exams] takes on heightened sensitivity" (Cohen et al. 1988). And unlike eye exams, some women may find the standard gynecologic exam to be "disempowering, abusive, and humiliating" (Bates et al. 2011, p. 652). The American College of Physician's clinical practice guideline also acknowledges the uniqueness of pelvic exams, noting how women may experience "fear, anxiety, embarrassment, pain, and discomfort" (Qaseem et al. 2014, p. 68) during a pelvic exam. Men may also express embarrassment (Myers et al. 1996), shame (Consedine et al. 2007), and mistrust (Robinson 1996) during rectal exams, and there is no literature relating to women's or men's shame or embarrassment during eye exams. These responses to intimate exams contribute to lower rates of doctor visits which may predispose patients to poorer health in the long term.

As discussed, women with a history of sexual violence (along with patients with sexual and gender minority status and other groups described earlier) are even more likely to experience distress during intimate exams. Ubel stated, "We don't see a pelvic exam as having any sexual content at all, but that's not how other people perceive it" (Goldstein 2003). "There's no way a physician would ever equate a pelvic exam with rape-there is no rape content to it. But the fact that someone else perceives it that way makes it important" (Goldstein 2003). An unconsented intimate exam may feel like a sexual violation to patients.

When medical school administrations consider the values they wish to promote in the next generation of physicians, they must consider patient perception. Even if some physicians and institutions have ambivalence over explicit consent for intimate procedures, patients, the general public, and others have repeatedly issued calls for explicit consent. Surveys indicate that women are unanimous in their stance against unconsented intimate exams by students when anesthetized (Bibby et al. 1988), and this is another signal that the medical community cannot gloss over this issue by, as an example, comparing the uterus to the eye.

Even though a patient's body may be "naked on a brightly lit table for all to see" (Friesen 2018a, p. 299) during a procedure, the patient should still have 
rights to decide what happens to their body. In a recent discussion forum about UIEs, a student wrote:

As a female student not yet in the medical field, I am disturbed to hear that by consenting to surgery, I risk having someone literally in my vagina without consent for purposes that benefit only [the students], and not me. Are patients really viewed as a teaching tool rather than a human being? That I will be splayed and sliced during the procedure doesn't mean that additional indignities are acceptable. It's still my vagina, even if I am naked and unconscious. I didn't lend it to anyone to practice techniques (Friesen 2018a, p. 300).

When people repeatedly express a fervent and heartfelt preference for how their bodies are treated, and this preference differs from physicians' perceptions, physicians should carefully examine why their perceptions should prevail. Clearly, standard consent forms fail to give patients the granularity they need to properly consent.

\section{Unconsented Exams are Infrequent}

Some argue that explicit consent is unnecessary because UIEs rarely occur. However, Greger, a physician who has researched unconsented exams, stated, "If they have five medical students on an OB-GYN rotation, they aren't going to let one do it and not the other four... I never heard of anyone out of some kind of respect for the patient just limiting it to a few" (Goldstein 2003). And, following recent discussions with concerned medical students, Caplan agreed: "Sometimes, more than one student [on their gynecology rotation] will practice the exam, with many sets of gloved fingers in the patient's vagina without their knowledge" (Caplan 2018a). Even if physicians rarely practice or condone UIEs, some do, and when institutions fail to enact explicit consent on the grounds that UIEs are infrequent, they send a message to the public that the affected patients' experiences are unimportant. Institutions should not fear that explicit consent will look badly on them. Instead they should view them as necessary protections for patients and know that patients are then more likely to relax in a clinical setting because of the policy's existence. In sum, the most common arguments in favor of the status quo generally lack substance and should not pose barriers to explicit consent.

\section{Discussion}

Critics of explicit consent point to perceived harms of intimate exams but fail to mention harms of continuing the status quo. Critics may argue that patients are not harmed if they are anesthetized (and therefore unable to remember the procedure), and perhaps that is true. However, some patients have recently woken during an UIE under anesthesia. Ashley Weitz, a young woman in Utah, sought emergency care for uncontrolled vomiting and when the physician suggested a pelvic exam to rule out a sexually transmitted disease, she explicitly declined (Laird 2019). Weitz passed out from the sedative effects of Phenergan then "woke up screaming" (Laird 
2019) because the physician was performing a pelvic exam. A survivor of childhood sexual assault, Weitz was so traumatized by the unconsented exam that she became an advocate for a law banning the procedure in her home state. Weitz is not the only patient to wake during an intimate exam (McDermott and Johnson 2019), and the idea that patients are unaware under anesthesia is also increasingly refuted: a large U.S. multicenter study found that intra-anesthetic awareness occurs in nearly 2000 cases every year (Sebel et al. 2004). Patients with awareness during surgeries most commonly report "hearing conversations... But there are many cases where they report pain, paralysis and anxiety because of the fact that they feel helpless [and some] patients describe this situation as the worst experience they ever had in a hospital" (Kotsovolis and Komninos 2009). One compelling report is from an anesthesiologist who, as a patient, was aware of aspects of his urogenital surgical experience and found his experience "terrifying" (Peduto et al. 1994). Therefore, the assumption that patients are unaware of their unconsented exams may be questionable.

Other patients have been informed of an UIE after they woke from anesthesia. One such patient, a nurse, woke from her laparoscopic stomach surgery in 2017 to be informed by a medical student that her cervix seemed abnormal (Graham 2019). She, too, is a survivor of sexual assault and was so distraught by the procedure that she tried to bring charges but was told by local attorneys that state laws would not allow her to prosecute the hospital. Definitions of medical malpractice vary by state, but medical malpractice occurs when a physician fails to disclose the risks of a surgery, provides substandard medical care, and subsequently injures the patient (Bal 2009). This nurse-patient's sense of violation and trauma could be considered injurious to her and may therefore meet the definition of medical malpractice.

Unconsented intimate exams typically occur on unconscious patients. After all, few would ever perform an unconsented intimate exam on a conscious patient because such an act is far outside of ethical standards. And yet there are recent cases of unconsented intimate exams on conscious patients. In some of these cases, the patients denied consent before the procedure but felt too overwhelmed to advocate for themselves during the exam. One patient, a physician from Maryland, was at his oncologist's office for a manual rectal exam related to his recovery from prostate cancer.

'...The doctor turned to a med student and said, 'Why don't you go ahead.'

The student dug right in, caving to the intense pressure med students are placed under during training. Stern, himself a doctor, was flabbergasted-not only because the doctor hadn't asked permission, but because Stern had expressly denied the student's own request to perform the exam not 10 min prior.

"It was terrible," says Stern now. "I was awake. I'd said no. But the trainee went ahead anyway, and neither of them spent any time telling me why they thought it was useful. "A patient shouldn't have to meet the needs of the provider," Stern goes on. "If he decides it's appropriate to help, great. But he needs to be asked" (Redfearn and Cao 2004).

While some may say that the patient could have spoken up, consent "must be given freely [and] validity is easily threatened if the patient... is asked for consent at an inappropriate time or is coerced" (UK Department of Health 2009). 
In Stern's case, the consent process was poorly executed and failed to protect the patient. It is interesting to note that the patient was a physician, someone who would seemingly feel most empowered to refuse a student exam, and yet even he felt constrained by hierarchical dynamics.

Other cases of UIEs on conscious patients may occur when the patient is unaware of the exams. In one such case (Rees and Monrouxe 2011), a male patient was conscious but unaware of receiving sequential digital rectal exams by several students since a sheet divided him from the students. Two of the students discussed this case as part of a recent multicenter study on intimate exams. One of the students stated:

...they just had a queue of medical students doing a rectal examination he wasn't consented but... you don't have the confidence to say 'no' you just do it...I think there was [sic] about three or four medical students... [the patient] had no idea that we were there and... none of the theatre staff spoke to him about what was happening (Rees and Monrouxe 2011, p. 268).

These cases find their way into public dialogue. As cases of conscious and unconscious UIEs become more widely known, patients may suspect their physicians are failing to fully disclose what will happen to their bodies during an exam. The fear that physicians may not share a sense of primary responsibility for their patients' health and well-being can damage the relationship between the general public and the medical community and lead to legal protections.

Medical students can also experience harm when the status quo goes unchallenged. As discussed, Ubel's study found that medical students were less supportive of explicit consent after their obstetrics and gynecology clerkship. No other rotations were reported to have this effect on medical students.

...this decline [in valuing the importance of informed consent] is not gradual and inexorable, but instead... it is associated with specific experiences. Students who have completed obstetrics/gynecology clerkships place significantly less importance on seeking permission from women... (Ubel et al., 2003, p. 578).

This shift in perceptions of consent is an example of ethical erosion, an attrition of values, leading to decreased respect for the patient's autonomy and emotional detachment. This shift may be attributable to the hidden curriculum, described by Mahood (2011) as:

a socialization process [by which] norms and values transmitted to future physicians often undermine the formal messages of the declared curriculum... [it] consists of what is implicitly taught by example day to day, not the explicit teaching of lectures, grand rounds, and seminars. Students move from being open-minded to being closed-minded; from being intellectually curious to narrowly focusing on facts; from empathy to emotional detachment; from idealism to cynicism; and often from civility and caring to arrogance and irritability. This erosion of empathy and "vanquishing of virtue" is repeatedly documented in studies of physicians in training (p. 983). 
Feudtner et al. (1994) have examined ethical erosion of medical students during their clerkships. Nearly all of the affected students in their study overheard physicians disparage patients, and over half observed members of the clinical team engage in unethical behavior and felt that their own ethical principles suffered during their clerkship. When students are faced with ethical dilemmas, Feudtner et al. find that "for at least a sizable minority, exposures to... [ethical] dilemmas coincide with deterioration of the students' ethical self-identities" (1994, p. 677), and those who saw others participate in unethical behaviors were increasingly likely to do so themselves.

Ethical erosion is not the only ill effect on medical students when they perform UIEs on patients. The experience of some students may be more accurately described in terms of moral distress, a "response to a perceived conflict between what one is expected to do and what morality requires" (Weber 2016, p. 249). Moral distress may arise "when one knows the right thing to do, but institutional constraints make it nearly impossible to pursue the right course of action" (Jameton 1984, p. 6). Moral distress is a "uniquely painful phenomenon... frequently associated with feelings of being powerless" (Tigard 2019, p. 605), and it correlates with compassion fatigue and high turnover rates. When Shawn Barnes (2012) was still a medical student, he felt coerced by institutional constraints to perform unconsented pelvic exams. He stated:

For 3 weeks, four to five times a day, I was asked to, and did, perform pelvic examinations on anesthetized women, without specific consent, solely for the purpose of my education. [When] the patient was asleep, the attending or resident would ask me to perform a pelvic examination on the patient for educational purposes. To my shame, I obeyed... My medical education experience has reinforced the notion that the medical student should not question the practices of those above him or her. I was very conflicted about performing an act that I felt was unethical, but owing to both the culture of medicine and my own lack of courage, I did not immediately speak out against what I was asked to do by residents and attendings" (Barnes 2012, p. 941).

Barnes felt powerless at the time but later chose to take action and helped to pass Hawaii's law banning unconsented pelvic exams. Symptoms of moral distress can ensue after direct or indirect participation in ethically questionable behavior, so nurses and other members of the clinical team may also experience moral distress when they witness or facilitate UIEs. It is not unreasonable to assume that indirect participants of UIEs may also experience ethical erosion. Thus, a move to an explicit consent process is not only good for the patient; it is also beneficial for the health and well-being of students and practitioners because explicit consent safeguards them from ethical erosion and symptoms of moral distress. When institutions decline to make consent explicit, they are a complicit party to ethical erosion and moral distress. And when institutions embrace an explicit consent process, they signal to their students and practitioners that they care about their well-being and are willing to make a small policy change to protect students' and practitioners' mental health and well-being. 
In sum, the continuation of the status quo results in a series of harms. Harms may occur to patients who are aware of having received an UIE, to medical students who are directed to conduct these exams, and to other members of the clinical team who watch or facilitate the procedure. Harms may also occur to the fragile relationship between the public and the medical community.

\section{Recommendations}

While the process of explicit consent may seem to be a trivial, burdensome, and perhaps uncomfortable administrative task, failure to acquire explicit consent inevitably harms some number of students, clinicians, and patients-and tarnishes the medical community's relationship with the general public. When medical schools enact an explicit consent process, they demonstrate the institution's respect for the patient as the gatekeeper to their body, recognize medical students' worth, and bolster their relationship with the general public. As discussed, most unconsented intimate exams are for training purposes on anesthetized patients during urological and obstetrical/ gynecological clerkships, and some UIEs have occurred for diagnostic purposes, on conscious patients, or during abdominal procedures. A sufficient resolution therefore requires explicit, institution-wide policies and also requires time, a precious commodity in medicine. Mercurio (2018) reflects on the physician's daily challenges, stating:

We know what we ought to do. The right to informed consent or permission, for example, has important ethical underpinnings... Most often, we fall short of our ethical ideals not from lack of deliberation, poor analysis, or inadequate understanding of ethical principles. We fall short because of time (p. 3).

These words should be taken to heart. Even when time is constrained (and it always seems to be), physicians should not overlook or gloss over the consent process with the patient. Indeed, the aspect of time is a fundamental determinant of resolving the decades-long conflict of UIEs. We spend our time on what we value. Spending time on explicit consent sends a signal to all parties that a patient's permission is sacred and valued. The resolution to UIEs requires a multi-tiered response. First, institutions should develop an explicit consent policy for all intimate exams. Second, medical school curriculum should support the explicit consent policy. Third, institutions should consider top-down changes to bolster ethics across the organization.

\section{An Explicit Consent Policy}

Institutions should create an explicit consent policy for all intimate exams regardless of the patient's gender, whether the patient shall be conscious or anesthetized during the procedure, and whether the exam is diagnostic or for training purposes. If the patient is unconscious and in a medical crisis, physicians can presume consent if and only if the patient's condition is so dire that the intimate exam cannot wait until after the patient regains consciousness. Finally, a patient should not receive an 
intimate exam unless it is indicated for the patient's underlying medical condition; a patient should never fear having an unconsented intimate exam when they are, for example, undergoing a cardiac procedure. Additionally, some consent forms may include a vague reference to student participation but may not specify whether a student intends to conduct an extra exam explicitly for training purposes. The presence of students - and the intent of their exams — should be made explicit to the patient.

The design of the consent form matters. As discussed, some hospitals use a standard surgical consent form. These forms should be modified to document explicit consent for intimate exams. Consent forms are also sometimes in electronic form. While some patients may prefer this method (Winter et al. 2016), electronic consent should not replace the physician-patient conversation.

Policy logistics also matter. The explicit consent policy should be publicly available and easily accessible so that all interested parties, including legislators and members of the general public, can locate it and have confidence in its existence. Also, when institutions care about the intent of a policy, they invest in training and prioritize periodic evaluations. Periodic reviews which thoughtfully examine the successes and failures of the policy could be employed to gauge progress.

Communications between physician and medical student also matter. Students rarely witness the consent conversation between the attending and the patient, so they do not always know whether explicit consent was acquired. Efforts should be made to allow students to observe (and learn) the consent process when possible. When students are unaware of the consent conversation, and are subsequently told to perform a student exam, they worry they are on shaky ethical ground. Students may not feel empowered to directly ask their supervisor whether explicit consent was obtained; they do not wish to challenge their superior's actions. To clear the air, once the physician has completed the explicit consent process with the patient, the physician should unambiguously inform students that explicit consent was obtained. This brief conversation between physician and medical student seems like a banal administrative task but it will ameliorate students' misplaced guilt, shame, and distress and provide enormous relief to them. Spending time on these conversationsand including students in the conversation whenever possible-underscores the importance of consent to students and the rest of the clinical team.

\section{Enhancements to the Medical School Curriculum}

Enhancements to the medical school curriculum will better prepare students to perform intimate exams. As discussed, some medical schools already employ complementary methods to learn intimate exams. These methods should become standard in all medical schools to help students learn intimate exams before patient contact, and the GTA position could be expanded to include role-playing. And as mentioned earlier, experts such as sexual assault counselors should be consulted to learn how to best support the sizeable patient population with risk factors predisposing them to anxiety or discomfort during intimate exams. The inclusion of these expert voices honors the psychosocial complexities inherent to the patient population. 
As discussed, Ubel's survey (2003) found that the completion of the obstetrics/ gynecology clerkship is associated with students' decreased interest in informed consent - a trend not found after any other clerkship. Medical schools should examine the dynamics of this rotation to determine root causes and take action to reverse this trend. Medical training should also teach students how to have a consent discussion with patients. Informed consent is a learned skill that can improve with practice and (as discussed) should be incorporated in students' training experiences with GTAs. Mercurio (2009) wrote a thoughtful reflection to help physicians request access to the bodies of newly deceased children for training exams. His reflection demonstrated how the use of sensitive, respectful language can build rapport with patients and families and provided guidelines which give structure and confidence to physicians also wishing to have these conversations with families. Similarly, guidelines could be developed to help physicians request consent for intimate exams and give them confidence during these sensitive discussions.

Medical school curriculum should provide bioethics education to students before and during clerkship rotations. Discussing cases in advance-and paths to resolution-will bolster students' understanding of ethical principles, help them understand how to navigate ethical conflicts before they arise, and may build their confidence. Medical schools should also tell students where to turn when they experience an ethical conflict. Medical schools should offer multiple channels for students to seek counsel; these delicate conversations require rapport between student and mentor.

During their clerkships, students should have avenues to report an ethically challenging encounter. Some medical schools have anonymous upward feedback mechanisms. Medical schools could also consider allowing a student to opt out of a procedure on ethical grounds. Physicians may elect a conscientious objection when they face an ethical challenge, and medical students should also have the means to refrain from an ethically fraught procedure without repercussions. Student access to conscientious objection is not widely available but is offered in some U.S. institutions (Card 2012).

\section{Changes to Medical School Culture}

Even with enhanced medical school education, students may not feel at liberty to refrain from conducting an unconsented intimate exam for training purposes. In Coldicott's study (2003a, b), many students felt unable to refuse their supervisor's call to perform an UIE. Similar concerns were voiced by medical students in Rees and Monrouxe's open-ended survey about professionalism in medicine (2011). This study collected reflections from 200 medical students and when asked generally about ethically charged circumstances, many students elected to discuss their experience with unconsented prostate and vaginal exams. One student who performed an UIE remembers feeling "almost star struck that I was in theatre for the first time" (p. 266). Another student felt bowled over by their supervisor, stating, "he was quite an aggressive surgeon anyway so I just went and did [the unconsented intimate exam]" (p. 266). These examples highlight medical students' inability to voice their 
concerns within the institution. It makes sense then, that some students approach outsiders to talk about their conflicts.

It is insufficient to make medical students solely responsible for halting their participation in UIEs. Therefore, directors of clerkships, the dean of students, attending physicians, and residents should all make clear that they support explicit consent, that explicit consent is mandatory, and that they support a student's right to exercise and voice an ethical concern. Institutions could also declare that violations to explicit consent will be investigated and offenders will be held accountable.

Physicians must act impeccably to preserve their trust with patients; when they do not, they damage the good name of their profession. Physicians should remember that their actions impact how patients view other physicians, and even small incidences of mistrust create fissures that are hard-if not impossible-to heal. Thus, physicians and medical schools should be supportive of policies that protect students and patients from indiscriminate physician behavior and help restore trust between patients and physicians.

Ethics can also be integrated across all levels of the medical institution. A notable example of integrated ethics includes the model developed for the Veterans Health Administration (Fox et al. 2010). Fox's model consists of three functions which target ethics at "the level of decisions and actions;... the level of systems and processes; and...the level of environment and culture" (p. 8). Work by Callahan et al. (2002) can also be considered: they promote "three trust-building tactics: 'accountability, reliance, and aspiration'... These principles provide people and mechanisms to reach the outcome of an ethical organizational culture" (p. 201). For instance, they suggest an ombudsperson to build employees' trust in the institution's policies and standards and recommend principles to increase empathy and trust and thereby foster organizational integrity. Similarly, Lachman (2008) suggests an implementation of committees, forums, and procedures to amplify integrity, and Greene and Latting (2004) recommend the formation of an organizational ethics committee. While these scholars propose robust approaches to institutional ethics, even small organizational changes can have lasting and far-reaching effects. To bolster a sound ethical culture, institutions should review these studies to identify methods most suitable to their institution. In summary, recommendations include an explicit consent policy and enhancements to both medical school curriculum and institutional culture.

\section{Conclusion}

Conflicts between physicians and families are a part of the public conversation. The case of Ms. Jahi McMath, an example of a family's refusal to accept a brain death diagnosis, caught national attention (Truog et al. 2018). The McMath family did not feel heard by their practitioners when their repeated calls to curb Jahi's postsurgical loss of blood were disregarded-neglect believed to have caused her brain death - and the case is emblematic of widespread racial bias in medicine (Goodwin 2018). Other families may also experience treatment denial, such as vaccine-hesitant families who are denied pediatric care (Flanagan-Klygis et al. 2005). These fissures reflect the challenge of conveying complex medical information to families who 
may already mistrust the medical system and the fissures seem to be so far-reaching that some fear there is little hope for resolution. Other recent news stories have covered Larry Nassar's rampant abuse of his pediatric patients (Mountjoy 2019) and the Atlanta Journal-Constitution's investigation uncovering "more than 3500 cases of sexual misconduct by physicians across the nation since 1999" (Teegardin and Norder 2019, p. 1). Given these recent headlines, it comes as no surprise that news coverage on unconsented intimate exams could cause further discord between physicians and the general public.

Ethicists, legal scholars, nurses, and professional associations-along with some physicians and medical students-have voiced persistent and prolonged calls for change, and the dichotomy between societal and traditional medical views of intimate regions shows no signs of abatement. UIEs continue to receive attention in the media, and legislators continue to propose statutes-underscoring the sustainability of these concerns. However, most institutions still decline to implement an explicit consent policy.

We are reminded that the bodies in question belong to the patients, and patients are the rights-holders of their bodies. Asking for permission is a fundamental tenet of modern medicine. Without permission, touching intimate areas can feel like an assault, and may even meet the criteria for battery or malpractice. This paper demonstrates how the status quo leads to ethical erosion, moral distress, patient distress, and distrust by the general public. These ill effects are surely not intended by physicians whose goals are to heal and comfort patients. When Rhodes urges physicians to "seek trust and deserve it as their moral law, as their creed," she notes that physicians should "pay serious attention to the patient's view of what is good" (2001, p. 497). Formalizing explicit consent can therefore be an act of trust-building between patients and physicians and call attention to physicians' honorable commitment to patients' safety and well-being.

We encourage medical institutions to reexamine their stance towards intimate exams to restore trust, protect clinicians, medical students, and patients, and underscore the sacred right of a patient to decide what happens to their body. Finally, while institutional change can minimize future harms, consideration should be paid to healing the wounds of medical students, physicians, and patients who have already suffered from past harms related to their roles in unconsented intimate exams.

Acknowledgements The author wishes to thank Jason Eiseman and Jordan Jefferson at the Lillian Goldman Law Library at Yale Law School, and Patty Martin at the Bioethics Research Library, Kennedy Institute of Ethics, Georgetown University for their valuable support during the research process.

\section{References}

Adashi, E. Y. (2019, January 16). JAMA forum: Teaching pelvic examination under anesthesia without patient consent. https:/newsatjama.jama.com/2019/01/16/jama-forum-teaching-pelvic-examinatio n-under-anesthesia-without-patient-consent/. Accessed 13 Sept 2019.

AMA Council on Ethical and Judicial Affairs. (2001, March). Medical student involvement in patient care: Report of the Council on Ethical and Judicial Affairs. https://journalofethics.ama-assn.org/artic le/medical-student-involvement-patient-care-report-council-ethical-and-judicial-affairs/2001-03. 
American College of Obstetricians and Gynecologists. (2009). Professional responsibilities in Obstetric-Gynecologic Education. ACOG Committee Opinion 2007; 358, January 2007.

American College of Obstetricians and Gynecologists. (2018, October). The Utility of and Indications for Routine Pelvic Examination. Retrieved from https://www.acog.org/-/media/Committee-Opini ons/Committee-on-Gynecologic-Practice/co754.pdf?dmc=1\&ts=20181126T1256054221.

American Medical Association. (2016). Opinions on patient-physician relationships. AMA Code of Medical Ethics. Chicago: American Medical Association.

Annas, G. J., \& the Legal Advisors Committee, Concern for Dying. (1983). The right to refuse treatment: A model act. American Journal of Public Health, 73(8), 918-921.

Annas, G. J., Glantz, L. H., \& Katz, B. F. (1977). Informed consent to human experimentation: The subject's dilemma. Cambridge: Ballinger Publishing Co.

Association of American Medical Colleges. (2003, June 12). AAMC Statement on patient rights and medical training. https://www.aamc.org/newsroom/pressrel/2003/030612.htm.

Bagg, W., Adams, J., Anderson, L., Malpas, P., Pidgeon, G., Thorn, M., et al. (2015). Medical students and informed consent: A consensus statement prepared by the faculties of medical and health science of the universities of Auckland and Otago, chief medical officers of district health boards, New Zealand medical students' association and the medical council of New Zealand. The New Zealand Medical Journal (Online), 128(1414), 27.

Bal, B. S. (2009). An introduction to medical malpractice in the United States. Clinical Orthopaedics and Related Research, 467(2), 339-347.

Barnes, S. S. (2012). Practicing pelvic examinations by medical students on women under anesthesia: Why not ask first? Obstetrics and Gynecology, 120(4), 941-943.

Barnes, S. S. (2019, December 15). In support of SB 2578. https://www.capitol.hawaii.gov/session201 2/Testimony/SB2578_TESTIMONY_HTH_2-10-12.pdf. Accessed 10 Sept 2019.

Bates, C. K., Carroll, N., \& Potter, J. (2011). The challenging pelvic examination. Journal of General Internal Medicine, 26(6), 651-657.

Beauchamp, T. L. (2011). Informed consent: Its history, meaning, and present challenges. Cambridge Quarterly of Healthcare Ethics, 20(4), 515-523.

Bhangu, A. A. (2003). Please don't touch me there: The ethics of intimate examinations: Consent is crucial—but don't go too far, for students' and patients' sakes. BMJ, 326(7402), 1326.

Bibby, J., Boyd, N., Redman, C. W. E., \& Luesley, D. M. (1988). Consent for vaginal examination by students on anaesthetised patients. The Lancet, 332(8620), 1150.

Boyles, S. (2003, January 9). Intimate exams without patient consent. https:/www.webmd.com/ women/news/20030109/intimate-exams-without-patient-consent\#1. Accessed 13 Dec 2019.

Callahan, E. S., Dworkin, T. M., Fort, T. L., \& Schipani, C. A. (2002). Integrating trends in whistleblowing and corporate governance: Promoting organizational effectiveness, societal responsibility, and employee empowerment. American Business Law Journal, 40(1), 177-236.

Caplan, A. L. (2018a, May 2). Pelvic exams done on anesthetized women without consent: Still happening. https://www.medscape.com/viewarticle/894693. Accessed 3 Dec 2019.

Caplan, A. L. (2018b, May 25). Med students still do pelvic exams on women under anesthesia. https ://www.medscape.com/viewarticle/896847. Accessed 3 Jan 2020.

Card, R. F. (2012). Is there no alternative? Conscientious objection by medical students. Journal of Medical Ethics, 38(10), 602-604.

Center for Disease Control and Prevention. (2013, October 28). Prevalence of disability in the US I CDC. https://www.cdc.gov/ncbddd/disabilityandhealth/features/key-findings-community-preva lence.html. Accessed 15 Nov 2019.

Centers for Disease Control and Prevention. (2016). FastStats. https://www.cdc.gov/nchs/fastats/obesi ty-overweight.htm. Accessed 15 Nov 2019.

Chan, S. (2017, July 7). Forced rectal exam stirs ethics questions. https://cityroom.blogs.nytim es.com/2008/01/16/forced-rectal-exam-stirs-ethics-questions. Accessed 14 Nov 2019.

Cohen, D. L., Kessel, R. W., McCullough, L. B., Apostolides, A. Y., Heiderich, K. J., \& Alden, E. R. (1989). Pelvic examinations by medical students. American Journal of Obstetrics and Gynecology, 161(4), 1013-1014.

Cohen, D. L., McCullough, L. B., Kessel, R. W., Apostolides, A. Y., Heiderich, K. J., \& Alden, E. R. (1988). A national survey concerning the ethical aspects of informed consent and role of medical students. Journal of Medical Education, 63(11), 821-829. 
Coldicott, Y., Nesheim, B. I., MacDougall, J., Pope, C., \& Roberts, C. (2003a). The ethics of intimate examinations-Teaching tomorrow's doctors. Commentary: Respecting the patient's integrity is the key. Commentary: Teaching pelvic examination-Putting the patient first. BMJ, 326(7380), 97-101.

Coldicott, Y., Pope, C., \& Roberts, C. (2003b). The ethics of intimate examinations-Teaching tomorrow's doctors (Education and debate). British Medical Journal, 326(7380), 97.

Consedine, N. S., Horton, D., Ungar, T., Joe, A. K., Ramirez, P., \& Borrell, L. (2007). Fear, knowledge, and efficacy beliefs differentially predict the frequency of digital rectal examination versus prostate specific antigen screening in ethnically diverse samples of older men. American Journal of Men's Health, 1(1), 29-43.

Department of Health. (2009). Reference guide to consent for examination or treatment, second edition. https://assets.publishing.service.gov.uk/government/uploads/system/uploads/attachment_data/ file/138296/dh_103653____.pdf.

Family Equality Council. (2017). LGBTQ family fact sheet. https://www2.census.gov/cac/nac/meeti ngs/2017-11/LGBTQ-families-factsheet.pdf.

Feudtner, C., Christakis, D. A., \& Christakis, N. A. (1994). Do clinical clerks suffer ethical erosion? Students' perceptions of their ethical environment and personal development. Academic Medicine: Journal of the Association of American Medical Colleges, 69(8), 670-679.

Flanagan-Klygis, E. A., Sharp, L., \& Frader, J. E. (2005). Dismissing the family who refuses vaccines: A study of pediatrician attitudes. Archives of Pediatrics and Adolescent Medicine, 159(10), 929-934.

Fox, E., Bottrell, M. M., Berkowitz, K. A., Chanko, B. L., Foglia, M. B., \& Pearlman, R. A. (2010). IntegratedEthics: An innovative program to improve ethics quality in health care. Innovation Journal, 15(2), 1-36.

Friesen, P. (2018a). Educational pelvic exams on anesthetized women: Why consent matters. Bioethics, 32(5), 298-307.

Friesen, P. (2018b, October 30). Why are pelvic exams on unconscious, unconsenting women still part of medical training? https://slate.com/technology/2018/10/pelvic-exams-unconscious-women-medicaltraining-consent.html. Accessed 13 Nov 2019.

Goldstein, A. (2003, May 10). Practice vs. privacy on pelvic exams. https://www.washingtonpost.com/ archive/politics/2003/05/10/practice-vs-privacy-on-pelvic-exams/4e9185c4-4b4c-4d6a-a132-b21b8 471da58/. Accessed 3 Nov 2019.

Goodwin, M. (2018). Revisiting death: Implicit bias and the case of Jahi McMath. Hastings Center Report, 48(S4), S77-S80.

Graham, J. (2019, January 1). The ethics of pelvic exams performed on anesthetized women without their knowledge. https://www.deseret.com/2019/1/2/20662352/the-ethics-of-pelvic-exams-performed-onanesthetized-women-without-their-knowledge. Accessed 13 Nov 2019.

Greene, A. D., \& Latting, J. K. (2004). Whistle-blowing as a form of advocacy: Guidelines for the practitioner and the organization. Social Work, 49(2), 219-230.

Jameton, A. (1984). Nursing practice: The ethical issues. Englewood Cliffs, NJ: Prentice-Hall.

Kelly, H. (2019, October 24). Pelvic exams are being performed on unconscious women without consent. https://www.vice.com/en_uk/article/59njez/nonconsensual-pelvic-exams-performed-unconsciou s-women. Accessed 13 Nov 2019.

Kotsovolis, G., \& Komninos, G. (2009). Awareness during anesthesia: How sure can we be that the patient is sleeping indeed? Hippokratia, 13(2), 83.

Lachman, V. D. (2008). Whistleblowing: Role of organizational culture in prevention and management. Medsurg Nursing, 17(4), 265-267.

Laird, L. (2019, September 1). Pelvic exams performed without patients' permission spur new legislation. http://www.abajournal.com/magazine/article/examined-while-unconscious. Accessed 13 Jan 2020.

Liu, K. E., Dunn, J. S., Robertson, D., Chamberlain, S., Shapiro, J., Akhtar, S. S., et al. (2010). Pelvic examinations by medical students. Journal of Obstetrics and Gynaecology Canada, 32(9), 872-874.

Mahood, S. C. (2011). Medical education: Beware the hidden curriculum. Canadian Family Physician, 57(9), 983-985.

Mcdermott, J. (2019, May 12). States seek explicit patient consent for pelvic exams. https://apnews.com/ c309d388b10b4fe582753e3b1f768f94. Accessed 13 Nov 2019.

Mercurio, M. R. (2009). Teaching intubation with cadavers: Generosity at a time of loss. Hastings Center Report, 39(4), 7-8.

Mercurio, M. (2018). Time is ethics. Hastings Center Report, 48(1), 3-4.

Mountjoy, M. (2019). 'Only by speaking out can we create lasting change': What can we learn from the Dr Larry Nassar tragedy? British Journal of Sports Medicine, 53(1), 57-60. 
Myers, R. E., Wolf, T. A., McKee, L., McGrory, G., Burgh, D. Y., Nelson, G., et al. (1996). Factors associated with intention to undergo annual prostate cancer screening among African American men in Philadelphia. Cancer: Interdisciplinary International Journal of the American Cancer Society, 78(3), 471-479.

National Sexual Violence Resource Center. (2018). Get statistics. https://www.nsvrc.org/node/4737. Accessed 15 Nov 2019.

Nelson, E. (2016). Teaching the female breast and pelvic exam: A student-and patient-centered approach. MedEdPORTAL. https://doi.org/10.15766/mep_2374-8265.10399.

Peduto, V. A., Silvetti, L., \& Piga, M. (1994). An anesthetized anesthesiologist tells his experience of waking up accidentally during the operation. Minerva Anestesiologica, 60(1-2), 1-5.

Picard, A. (2018, May 12). Time to end pelvic exams done without consent. https://www.theglobean dmail.com/life/health-and-fitness/time-to-end-pelvic-exams-done-without-consent/article4325965/. Accessed 13 Jan 2020.

Potter, J., Fessler, D., Huang, G., Baker, J., Dearborn, H., \& Libman, H. (2015). Challenging pelvic exam. MedEdPORTAL. https://doi.org/10.15766/mep_2374-8265.10256.

Qaseem, A., Humphrey, L. L., Harris, R., Starkey, M., \& Denberg, T. D. (2014). Screening pelvic examination in adult women: A clinical practice guideline from the American College of Physicians. Annals of Internal Medicine, 161(1), 67-72.

Qin, J., Saraiya, M., Martinez, G., \& Sawaya, G. F. (2020). Prevalence of potentially unnecessary bimanual pelvic examinations and Papanicolaou tests among adolescent girls and young women aged 15-20 years in the United States. JAMA Internal Medicine, 180(2), 274-280.

Redfearn, S. \& Cao, H. (2004, July 6). Are med students practicing on you? https://www.menshealth .com/health/a19528004/are-med-students-practicing-on-you/. Accessed 13 Sept 2019.

Rees, C. E., \& Monrouxe, L. V. (2011). Medical students learning intimate examinations without valid consent: A multicentre study. Medical Education, 45(3), 261-272.

Rhodes, R. (2001). Understanding the trusted doctor and constructing a theory of bioethics. Theoretical Medicine and Bioethics, 22(6), 493-504.

Robinson, S. B., Ashley, M., \& Haynes, M. A. (1996). Attitudes of African Americans regarding screening for prostate cancer. Journal of the National Medical Association, 88(4), 241.

Royal College of Obstetricians and Gynaecologists (2015). Obtaining valid consent. Clinical Governance Advice No. 6.

Schniederjan, S., \& Donovan, G. K. (2005). Ethics versus education: Pelvic exams on anesthetized women. Journal: Oklahoma State Medical Association, 98(8), 386-388.

Sebel, P. S., Bowdle, T. A., Ghoneim, M. M., Rampil, I. J., Padilla, R. E., Gan, T. J., et al. (2004). The incidence of awareness during anesthesia: A multicenter United States study. Anesthesia and Analgesia, 99(3), 833-839.

Teegardin, C., \& Norder, L. (2019). Abusive doctors: How the Atlanta newspaper exposed a system that tolerates sexual misconduct by physicians. London: Taylor \& Francis.

The Local. (2015, October 29). France to ban vaginal exams without consent. https://www.thelocal. fr/20151029/france-bans-unconsented-vaginal-exams. Accessed 13 Sept 2019.

Tigard, D. W. (2019). The positive value of moral distress. Bioethics, 33(5), 601-608.

Truog, R. D., Berlinger, N., Zacharias, R. L., \& Solomon, M. Z. (2018). Brain death at fifty: Exploring consensus, controversy, and contexts. Hastings Center Report, 48(S4), S2-S5.

Tsai, J. (2019, June 24). Medical students regularly practice pelvic exams on unconscious patients. should they? https://www.elle.com/life-love/a28125604/nonconsensual-pelvic-exams-teachinghospitals/. Accessed 13 Nov 2019.

Ubel, P. A., Jepson, C., \& Silver-Isenstadt, A. (2003). Don't ask, don't tell: A change in medical student attitudes after obstetrics/gynecology clerkships toward seeking consent for pelvic examinations on an anesthetized patient. American Journal of Obstetrics and Gynecology, 188(2), 575-579.

US Preventive Services Task Force. (2017). Final recommendation statement. Gynecological conditions: Periodic screening with the pelvic examination. Rockville: US Preventive Services Task Force.

US Preventive Services Task Force. (2018). Final recommendation statement. Screening for prostate cancer. Rockville: US Preventive Services Task Force.

Wainberg, S., Wrigley, H., Fair, J., \& Ross, S. (2010). Teaching pelvic examinations under anaesthesia: what do women think? Journal of Obstetrics and Gynaecology Canada, 32(1), 49-53.

Walsh, K. (Ed.). (2013). Oxford textbook of medical education. Oxford: Oxford University Press.

Weber, E. (2016). Moral distress, workplace health, and intrinsic harm. Bioethics, 30(4), 244-250. 
Wilson, R. F. (2005). Autonomy suspended: Using female patients to teach intimate exams without their knowledge or consent. Journal of Health Care Law and Policy, 8(2), 240.

Winter, M., Kam, J., Nalavenkata, S., Hardy, E., Handmer, M., Ainsworth, H., et al. (2016). The use of portable video media vs standard verbal communication in the urological consent process: A multicentre, randomised controlled, crossover trial. BJU International, 118(5), 823-828.

Publisher's Note Springer Nature remains neutral with regard to jurisdictional claims in published maps and institutional affiliations. 